\title{
Cerebrovascular
}

Diseases

\section{8th European Stroke Conference}

Abstracts

Venice, Italy

April 7-10, 1999
Editor

M.G. Hennerici, Mannheim 


\section{KARGER}

S. Karger

Medical and Scientific Publishers

Basel $\cdot$ Freiburg $\cdot$ Paris $\cdot$ London

New York $\cdot$ New Delhi $\cdot$ Bangkok

Singapore $\cdot$ Tokyo $\cdot$ Sydney
Drug Dosage

The authors and the publisher have exerted every effort to ensure that drug selection and dosage set forth in this text are in accord with current recommendations and practice at the time of publication. However, in view of ongoing research, changes in government regulations, and the constant flow of information relating to drug therapy and drug reactions, the reader is urged to check the package insert for each drug for any change in indications and dosage and for added warnings and precauin in. This is partione in for ant whe tions. This is particuly impert when the recom tions. This is particuly important when the recor
All rights reserved.

No part of this publication may be translated into other languages, reproduced or utilized in any form or by any means, electronic or mechanical, including photocopying, recording, microcopying, or by any information storage and retrieval system, without permission in writing from the publisher or, in the case of photocopying, direct payment of a specified fee to the Copyright Clearance Center (see 'General Information').

(c) Copyright 1999 by S. Karger AG,

P.O. Box, CH-4009 Basel (Switzerland)

Printed in Switzerland on acid-free paper by

Reinhardt Druck, Basel

Reinhardt Druck, Base 


\section{Cerebrovascular \\ Diseases}

\section{Gian Luigi Lenzi, Rome}

Conference Chairman

\section{Committees}

\section{ESC Programme Committee}

Franz Aichner, Innsbruck

Julien Bogousslavsky, Lausanne

José M. Ferro, Lisboa

Michael G. Hennerici, Mannheim

Gian Luigi Lenzi, Rome

Jean-Marc Orgogozo, Bordeaux

Nils Gunnar Wahlgren, Stockholm

\section{Scientific Committee}

Franz Aichner, Austria

Pierre Amarenco, France

John Bamford, United Kingdom

Serge Blecic, Belgium

Julien Bogousslavsky, Switzerland

Nathan Bornstein, Israel

Marie-Germaine Bousser, France

Martin Brown, United Kingdom

Livia Candelise, Italy

Antonio Carolei, Italy

Laszlo Csiba, Hungary

Anna Czlonkowska, Poland

Jacques de Reuck, Belgium

Franz Fazekas, Austria

Valery Feigin, Russia

Jose M. Ferro, Portugal

Cesare Fieschi, Italy

Jan van Gijn, The Netherlands

Werner Hacke, Germany

Michael G. Hennerici, Germany

Domenico Inzitari, Italy

Markku Kaste, Finland

Peter Koudstaal, The Netherlands

Wolfgang Kuschinsky, Germany

Gian Luigi Lenzi, Italy

Jan Lodder, The Netherlands

Jean-Louis Mas, France

Jordi Matias-Guiu, Spain

Heinrich Mattle, Switzerland

Bo Norrving, Sweden

Tom Skyhoj Olsen, Denmark

Jean-Marc Orgogozo, France

Gazi Özdemir, Turkey

E. Berndt Ringelstein, Germany

David Russell, Norway

Peter Sandercock, United Kingdom

Nils Gunnar Wahlgren, Sweden

Charles Warlow, United Kingdom 


\section{Cerebrovascular \\ Diseases}

\section{Sponsors}

The 8th European Stroke Conference is supported by

Main Sponsors

Astra

Ministero degli Affari Esteri

Direzione Generale delle Relazioni Culturali

Comune di Venezia

Società Italiana di Neurologia

This Conference is endorsed by the American Academy of Neurology. Academy members and all interested neurologists are encouraged to attend.

Free registration and lodgings for selected students from East Europe and underdeveloped countries have been made possible by a grant from the Cultural Relation Department of the Italian Foreign Office.
BASF Pharma/KNOLL AG

Boehringer Ingelheim

Bristol-Myers Squibb

GlaxoWellcome Research \& Development

Janssen Pharmaceutica

Sanofi

UCB Pharma
Sponsors

Assicurazioni Generali

Bayer AG

Chiesi Farmaceutici

Ely Lilly Italia

Gruppo Ligabue

LILLY France

PARKE-DAVIS

Ravizza Farmaceutici

Roerig Farmaceutici Italiana

sigma-tau

Wyeth Lederle 


\section{Cerebrovascular Diseases}

\section{Scientific Programme}

\begin{tabular}{|c|c|c|}
\hline \multicolumn{3}{|c|}{ Thursday, April 8, 1999} \\
\hline 8:30-9:00 & Opening Welcome Address & G. Lenzi, M.G. Hennerici \\
\hline \multirow[t]{5}{*}{ 9:00-10:30 } & $\begin{array}{l}\text { Symposium I: New Aspects of Acute Stroke Treatment as } \\
\text { Evidence from Pre-Clinic and Experimental Studies }\end{array}$ & $\begin{array}{l}\text { Chairpersons: J.C. Baron (France), } \\
\text { U. Dirnagl (Germany) }\end{array}$ \\
\hline & $\begin{array}{l}\text { Excitotoxicity, Inflammation and Apoptosis: The Cascade } \\
\text { of Cerebral Ischemic Damage }\end{array}$ & U. Dirnagl (Germany) \\
\hline & $\begin{array}{l}\text { Experimental Reperfusion Injury: Still an Issue for Stroke } \\
\text { Therapy? }\end{array}$ & P. Lindsberg (Finland) \\
\hline & $\begin{array}{l}\text { Microcirculatory and Microvascular Events after } \\
\text { Temporary Cerebral Ischemia }\end{array}$ & G.J. del Zoppo (USA) \\
\hline & $\begin{array}{l}\text { Metabolic and Cellular Derangement after MCA Occlusion } \\
\text { in the Baboon: Core versus Penumbra }\end{array}$ & A.R. Young (France) \\
\hline 10:30-11:00 & Coffee/Tea Break & \\
\hline \multirow[t]{9}{*}{$11: 00-12: 20$} & Platform Session I (Clinical/General Stroke) & $\begin{array}{l}\text { Chairpersons: J.-M. Orgogozo (France), } \\
\text { A. Czlonkowska (Poland) }\end{array}$ \\
\hline & $\begin{array}{l}\text { Infection in Acute Stroke. Type, Frequency and the } \\
\text { Relation to Stroke Severity, Mortality and Outcome. } \\
\text { The Copenhagen Stroke Study (COST) }\end{array}$ & L.P. Kammersgaard, et al. (Denmark) \\
\hline & Anti-hsp Antibodies in Ischemic Stroke & A. Czlonkowska, et al. (Poland) \\
\hline & $\begin{array}{l}\text { Acute 24-Hour Systolic Blood Pressure Predicts Longterm } \\
\text { Stroke Outcome }\end{array}$ & T. Robinson, et al. (UK) \\
\hline & Dementia Immediately and Three Months after Acute Stroke & A. Tsiskaridze, et al. (Georgia) \\
\hline & $\begin{array}{l}\text { Stroke in People Practising Sports. Frequency and } \\
\text { Etiology }\end{array}$ & S. Blecic, et al. (Belgium) \\
\hline & Factors Associated with Sleep Apnea in Stroke Patients & O. Sandberg, et al. (Sweden) \\
\hline & $\begin{array}{l}\text { Prevalence of Symptomatic Intercranial Aneurysm and } \\
\text { Ischaemic Stroke in Pseudoxanthoma Elasticum }\end{array}$ & $\begin{array}{l}\text { J.S.P. van den Berg, et al. } \\
\text { (The Netherlands) }\end{array}$ \\
\hline & $\begin{array}{l}\text { The Lausanne Emotion in Stroke Study. Emotional } \\
\text { Behavior in Acute Stroke }\end{array}$ & F. Ghika-Schmid, et al. (Switzerland) \\
\hline $12: 20-13: 00$ & $\begin{array}{l}\text { Invited Lecture I } \\
\text { Zinc and Ischemic Brain Injury }\end{array}$ & $\begin{array}{l}\text { Chairperson: W. Kuschinsky (Germany) } \\
\text { Lecturer: D. Choi (USA) }\end{array}$ \\
\hline 13:00-15:00 & Lunch & \\
\hline 13:00-15:00 & Poster Session I & $\begin{array}{l}\text { Chairpersons: V. Feigin (Russia), } \\
\text { L. Caplan (USA), M. Daffertshofer } \\
\text { (Germany) }\end{array}$ \\
\hline \multirow[t]{3}{*}{ 15:00-16:00 } & Platform Session II A (Neuroimaging) & $\begin{array}{l}\text { Chairpersons: F. Fazekas (Austria), } \\
\text { S. Warach (USA) }\end{array}$ \\
\hline & Time Course of Lesion Development in Hyperacute Stroke & S. Behrens, et al. (Germany) \\
\hline & $\begin{array}{l}\text { Relation of Perfusion-Weighted Magnetic Resonance } \\
\text { Imaging (MRI) and Clinical Outcome in Patients with } \\
\text { Ischemic Stroke }\end{array}$ & C. Chaves, et al. (USA) \\
\hline
\end{tabular}

\section{KARGER}

(C) 1999 S. Karger AG, Basel

Fax+4161306 1234

E-Mail karger@karger.ch

www.karger.com 


\begin{tabular}{|c|c|c|}
\hline & $\begin{array}{l}\text { Asymptomatic New Ischaemic Lesions on Serial MRI } \\
\text { Scans }\end{array}$ & P.A. Holmes, et al. (UK) \\
\hline & $\begin{array}{l}\text { Cortical Extension of the Infarct in Patients with Initial } \\
\text { Subcortical Damage Can Be a Useful Endpoint for Clinical } \\
\text { Trials in Acute Ischemic Stroke }\end{array}$ & M. Fiorelli, et al. (Italy) \\
\hline & $\begin{array}{l}\text { Do Early CT Signs Reflect only Pre-Infarction? } \\
\text { A Comparative CT-DWI Study }\end{array}$ & A. Jaillard, et al. (France) \\
\hline & $\begin{array}{l}\text { A Comparison of Electron-Beam-Tomography (EBT) and } \\
\text { Transesophageal Echocardiography (TEE) for Diagnosis of } \\
\text { Aortic Atheroma in Stroke Patients }\end{array}$ & M. Aichinger-Steiner, et al. (Austria) \\
\hline \multirow[t]{7}{*}{ 15:00-16:00 } & Platform Session II B (Experimental) & $\begin{array}{l}\text { Chairpersons: K. Overgaard (Denmark), } \\
\text { A. Davalos (Spain) }\end{array}$ \\
\hline & $\begin{array}{l}\text { Extracellular Shifts of GABA, Adenosine and lon Activities } \\
\text { in Gray and White Matter during Global Cerebral Ischemia } \\
\text { in Cats }\end{array}$ & R. Graf, et al. (Germany) \\
\hline & $\begin{array}{l}\text { Excitotoxic Effect of High Frequency Stimulation during } \\
\text { Hypoxia in Hippocampal Slices: Neuroprotection by AP-5, } \\
\text { a Selective NMDA Receptor Antagonist }\end{array}$ & G. Capocchi, et al. (Italy) \\
\hline & $\begin{array}{l}\text { Neuroprotective Efficacy of the Novel 5-HT 1A Receptor } \\
\text { Agonist BAYx3702 in Rat Models of Permanent and } \\
\text { Transient Focal Cerebral Ischemia }\end{array}$ & E. Horváth, et al. (Germany) \\
\hline & $\begin{array}{l}\text { NXY-059: 5-Hour Treatment Window in a Transient Focal } \\
\text { Stroke Model }\end{array}$ & K. Maples, et al. (USA) \\
\hline & $\begin{array}{l}\text { Plasma Excitatory Amino Acid Concentrations after } \\
\text { Permanent Middle Cerebral Artery Occlusion }\end{array}$ & A. Davalos, et al. (Spain) \\
\hline & $\begin{array}{l}\text { Corticotrophin-Releasing Factor Increases Cerebral Blood } \\
\text { Flow in the Rat: A Novel Modulator System of Cerebral } \\
\text { Blood Flow? }\end{array}$ & M. De Michele, et al. (Italy) \\
\hline $16: 00-16: 30$ & Coffee/Tea Break & \\
\hline \multirow[t]{8}{*}{ 16:30-18:00 } & Platform Session III A (Vascular Physiology/CBF) & $\begin{array}{l}\text { Chairpersons: R.W. Baumgartner } \\
\text { (Switzerland), W.D. Heiss (Germany) }\end{array}$ \\
\hline & $\begin{array}{l}\text { Dorsomedial Origin of the Internal Carotid Artery as a } \\
\text { Risk Factor for Early and Advanced Carotid } \\
\text { Atherosclerosis }\end{array}$ & M. Sitzer, et al. (Germany) \\
\hline & $\begin{array}{l}\text { Coronary-Type Plaque Instability in the Carotid Artery and } \\
\text { Its Detection by Ultrasound }\end{array}$ & G.A. Lammie, et al. (UK) \\
\hline & $\begin{array}{l}\text { Atherosclerotic Lesions in the Aortic Arch and in the } \\
\text { Carotid Arteries in Patients with Stroke }\end{array}$ & T. Mendel, et al. (Poland) \\
\hline & $\begin{array}{l}\text { Intracranial Arterial Stenosis in Patients with Acute } \\
\text { Ischaemic Cerebrovascular Disease: A Transcranial } \\
\text { Doppler Study }\end{array}$ & T. Segura, et al. (Spain) \\
\hline & $\begin{array}{l}\text { Transcranial Doppler Diagnosis of Delayed Ischaemic } \\
\text { Neurological Deficit following Subarachnoid } \\
\text { Haemorrhage-How Valid a Technique? }\end{array}$ & P.S. Minhas, et al. (UK) \\
\hline & $\begin{array}{l}\text { Cerebral Vasomotor Reactivity and Cholesterol and } \\
\text { High-Density Lipoprotein (HDL) in the Elderly }\end{array}$ & S.L.M. Bakker, et al. (The Netherlands) \\
\hline & $\begin{array}{l}\text { Dynamic Cerebral Autoregulation Varies According to } \\
\text { Blood Pressure Stimulus }\end{array}$ & S.L. Dawson, et al. (UK) \\
\hline
\end{tabular}




\begin{tabular}{|c|c|c|}
\hline & $\begin{array}{l}\text { Preferential Sensitivity of Borderzone Areas to Decreases } \\
\text { in Cerebral Blood Flow Demonstrated by Dynamic } \\
\text { Susceptibility Contrast Enhanced MRI }\end{array}$ & A. Jackson, et al. (UK) \\
\hline & $\begin{array}{l}\text { Quantification of Collateralisation in Unilateral Stenotic } \\
\text { Internal Carotid Artery Disease Using 2D Cine Phase- } \\
\text { Contrast MR-Imaging }\end{array}$ & A. Schwartz, et al. (Germany) \\
\hline \multirow[t]{10}{*}{ 16:30-18:00 } & Platform Session III B (Epidemiology and Risk Factors) & $\begin{array}{l}\text { Chairpersons: M. Brown (UK), } \\
\text { J. Lodder (The Netherlands) }\end{array}$ \\
\hline & $\begin{array}{l}\text { Marked Improvement since } 1985 \text { in Short-Term and } \\
\text { Long-Term Survival after Stroke }\end{array}$ & M. Peltonen, et al. (Sweden) \\
\hline & $\begin{array}{l}\text { Social Patterning of Cardiovascular Disease: A Comparison } \\
\text { between Stroke and Myocardial Infarction }\end{array}$ & M. Peltonen, et al. (Sweden) \\
\hline & $\begin{array}{l}\text { Homocysteine in Cerebrovascular Disease: Role as an } \\
\text { Independent Risk Factor for Subcortical Vascular } \\
\text { Encephalopathy }\end{array}$ & K. Faßbender, et al. (Germany) \\
\hline & $\begin{array}{l}\text { Higher Dietary Intake of Vitamin B6 Is Associated with a } \\
\text { Lower Risk of Ischemic Stroke }\end{array}$ & B.S. Jacobs, et al. (USA) \\
\hline & $\begin{array}{l}\text { The Platelet Collagen Receptor GP Ia C807T Polymorphism } \\
\text { Is an Independent Risk Factor in Young Stroke Patients }\end{array}$ & C. Spitzer, et al. (Germany) \\
\hline & $\begin{array}{l}\text { Left Ventricular Geometric Patterns and the Risk of } \\
\text { Ischemic Stroke }\end{array}$ & M.R. Di Tullio, et al. (USA) \\
\hline & $\begin{array}{l}\text { Socioeconomic Status and Ischaemic Stroke Events in } \\
\text { Finland }\end{array}$ & P. Immonen-Räihä, et al. (Finland) \\
\hline & $\begin{array}{l}\text { The Incidence and } 1 \text { Year Case-Fatality of Stroke in } 3 \text { Areas } \\
\text { of Europe in } 1995\end{array}$ & R. Dundas, et al. (UK) \\
\hline & $\begin{array}{l}\text { Cerebral Small Vessel Disease Compared with Large } \\
\text { Vessel Atherosclerosis: Genetic and Vascular Risk Factors }\end{array}$ & V.I.H. Kwa, et al. (The Netherlands) \\
\hline \multicolumn{3}{|c|}{ Friday, April 9, 1999} \\
\hline \multirow[t]{5}{*}{$8: 30-10: 30$} & Symposium II: Stroke and Prevention & $\begin{array}{l}\text { Chairpersons: M.G. Hennerici (Germany), } \\
\text { G. Hankey (Australia) }\end{array}$ \\
\hline & Intracranial Aneurysms and Genetics & A. Ronkainen (Finland) \\
\hline & $\begin{array}{l}\text { Genetic Factors that Influence Stroke Severity, Recovery } \\
\text { and Outcome }\end{array}$ & A. Catto (UK) \\
\hline & New Targets for Stroke Prevention & S. Davis (Australia) \\
\hline & $\begin{array}{l}\text { New Antiplatelet Strategies in Vascular Medicine and } \\
\text { Cardiology }\end{array}$ & J. Beer (Switzerland) \\
\hline $10: 30-11: 00$ & Coffee/Tea Break & \\
\hline 11:00-11:30 & $\begin{array}{l}\text { Invited Lecture II } \\
\text { New Insights in Moya-Moya Disease }\end{array}$ & $\begin{array}{l}\text { Chairperson: J. v Gijn (The Netherlands) } \\
\text { Lecturer: Y.Yonekawa (Switzerland) }\end{array}$ \\
\hline \multirow[t]{4}{*}{$11: 30-13: 00$} & Platform Session IV (Prevention) & $\begin{array}{l}\text { Chairpersons: J. Ferro (Portugal), } \\
\text { B. Norrving (Sweden) }\end{array}$ \\
\hline & $\begin{array}{l}\text { Plasma Fibrinogen Levels and Ischaemic Stroke: Data from } \\
\text { the Bezafibrate Infarction Prevention (BIP) Study }\end{array}$ & D. Tanne, et al. (Israel) \\
\hline & $\begin{array}{l}\text { Dual Chamber Paced Patients - An Underestimated Risk } \\
\text { Group for Stroke? }\end{array}$ & F. Saborowski, et al. (Germany) \\
\hline & $\begin{array}{l}\text { Stroke and Atrial Fibrillation: Was Stroke Prevention } \\
\text { Appropriate Beforehand? }\end{array}$ & D. Deplanque, et al. (France) \\
\hline
\end{tabular}




\begin{tabular}{|c|c|c|}
\hline & $\begin{array}{l}\text { Low Risk of Ischaemic Stroke in Patients with } \\
\text { Symptomatic Carotid Near-Occlusion: Implications for } \\
\text { Imaging and Treatment }\end{array}$ & P. Rothwell, et al. (UK) \\
\hline & $\begin{array}{l}\text { The Cost Effectiveness of Clopidogrel and the } \\
\text { Combination of Aspirin and Dipyridamole in Stroke } \\
\text { Prevention }\end{array}$ & J.R. Overell, et al. (UK) \\
\hline & $\begin{array}{l}\text { The Asymptomatic Carotid Stenosis Study (ACSS): } \\
\text { Differences in the Time Course of Risk of Ischaemic Stroke } \\
\text { Distal to Symptomatic and Asymptomatic Carotid } \\
\text { Stenoses }\end{array}$ & P. Rothwell, et al. (UK) \\
\hline & $\begin{array}{l}\text { Carotid and Vertebral Artery Transluminal Angioplasty } \\
\text { Study (CAVATAS): } 3 \text { Year Outcome Data }\end{array}$ & M.M. Brown, et al. (UK) \\
\hline & $\begin{array}{l}\text { Low-Dose Heparin Stroke Prevention Study: Preliminary } \\
\text { Efficacy Analysis }\end{array}$ & G.G. Neri Serneri, et al. (Italy) \\
\hline 13:00-14:30 & Lunch & \\
\hline 13:00-14:30 & Poster Session II & $\begin{array}{l}\text { Chairpersons: W. Hacke (Germany), } \\
\text { A. Carolei (Italy), N. Bornstein (Israel) }\end{array}$ \\
\hline \multirow[t]{5}{*}{$14: 30-16: 30$} & $\begin{array}{l}\text { Symposium III: Obstacles in Translational Stroke } \\
\text { Research from Animal to Man }\end{array}$ & $\begin{array}{l}\text { Chairpersons: M.-G.Bousser (France), } \\
\text { M.A. Moskowitz (USA) }\end{array}$ \\
\hline & Does the Human Condition Reflect Animal Models? & A. Boisson (France) \\
\hline & What Have We Learnt from Clinical Trials? & N.G. Wahlgren (Sweden) \\
\hline & Targeting Stroke Recovery & S. Finkelstein (USA) \\
\hline & Promising Therapeutic Targets & M.A. Moskowitz (USA) \\
\hline 16:30-17:00 & Coffee/Tea Break & \\
\hline \multirow[t]{7}{*}{ 17:00-18:00 } & Platform Session V A (Recovery/Physical Therapy) & $\begin{array}{l}\text { Chairpersons: P. Tonin (Italy), } \\
\text { V. Hömberg (Germany) }\end{array}$ \\
\hline & Stroke Unit Treatment - 10 Years Follow-Up & B. Indredavik, et al. (Norway) \\
\hline & $\begin{array}{l}\text { A Randomised Controlled Trial of Occupational Therapy } \\
\text { for Stroke Patients Not Admitted to Hospital }\end{array}$ & M.F. Walker, et al. (UK) \\
\hline & $\begin{array}{l}\text { Marked Reduction in Mortality } 5 \text { Years after Stroke Unit } \\
\text { Treatment }\end{array}$ & H.S. Jørgensen, et al.(Denmark) \\
\hline & $\begin{array}{l}\text { Dynamic Changes of Neuroplasticity in Stroke: A Serial } \\
\text { PET Study }\end{array}$ & G. Nelles, et al. (Germany) \\
\hline & $\begin{array}{l}\text { Randomised Controlled Trial of a Stroke Family Support } \\
\text { Organiser }\end{array}$ & J. Mant, et al. (UK) \\
\hline & $\begin{array}{l}\text { A Randomised Double Blind Placebo Controlled Trial of } \\
\text { Botulinum Toxin Treatment on the Disabling Effects of } \\
\text { Severe Arm Spasticity in Stroke }\end{array}$ & B.B. Bhakta, et al. (UK) \\
\hline \multirow[t]{4}{*}{ 17:00-18:00 } & Platform Session V B (Acute Treatment - Thrombolysis) & $\begin{array}{l}\text { Chairpersons: M. Kaste (Finland), } \\
\text { G. Donnan (Australia) }\end{array}$ \\
\hline & $\begin{array}{l}\text { Thrombolytic Therapy for Acute Ischaemic Stroke - } \\
\text { The Updated Cochrane Database of Systematic Reviews } \\
\text { Meta-Analysis }\end{array}$ & J.M. Wardlaw, et al. (UK) \\
\hline & $\begin{array}{l}\text { Post Approval Experience with Intravenous t-PA for } \\
\text { Treatment of Acute Stroke: A Phase IV Multicenter, } \\
\text { Prospective, Monitored Study }\end{array}$ & S.A. Hamilton, et al. (USA) \\
\hline & $\begin{array}{l}\text { Long-Term Outcome after Intravenous Thrombolysis of } \\
\text { Ischaemic Stroke }\end{array}$ & S. Schmülling, et al. (Germany) \\
\hline
\end{tabular}




\begin{tabular}{|c|c|c|}
\hline & $\begin{array}{l}\text { Tissue Plasminogen Activator in Acute Stroke Patients } \\
\text { with an Unruptured Brain Aneurysm }\end{array}$ & L. D’Olhaberriague, et al. (USA) \\
\hline & $\begin{array}{l}\text { How Many Patients Can Be Treated and Saved with } \\
\text { Thrombolysis? }\end{array}$ & S. Ricci, et al. (Italy) \\
\hline & $\begin{array}{l}\text { Pro-Urokinase in Acute Thromboembolic Stroke } \\
\text { (PROACT) Study: Results }\end{array}$ & A. Furlan, et al. (USA) \\
\hline 18:00-19:00 & $\begin{array}{l}\text { European Stroke Council/European Stroke Conference, } \\
\text { General Assembly }\end{array}$ & \\
\hline \multicolumn{3}{|c|}{ Saturday, April 10, 1999} \\
\hline \multirow[t]{6}{*}{$8.30-10.30$} & Symposium IV: Recovery after Acute Stroke & $\begin{array}{l}\text { Chairpersons: J. Bogousslavsky } \\
\text { (Switzerland), C. Fieschi (Italy) }\end{array}$ \\
\hline & $\begin{array}{l}\text { Biological Correlates of Early and Late Recovery after } \\
\text { Brain Damage }\end{array}$ & H.-J. Freund (Germany) \\
\hline & Pharmacological Enhancement of Stroke Recovery & M. Fisher (USA) \\
\hline & $\begin{array}{l}\text { Measures to Improve Recovery in the Acute Phase of } \\
\text { Stroke }\end{array}$ & P. Langhorne (UK) \\
\hline & Improvement of Aphasia and Neglect in Acute Stroke & J. Ferro (Portugal) \\
\hline & Motor Recovery after Stroke (Invited Lecture) & Lecturer: R.S.I. Frackowiak (UK) \\
\hline $10: 30-11: 00$ & Coffee/Tea Break & \\
\hline 10:50-11:00 & Awards & $\begin{array}{l}\text { Chairpersons: D. Easton (USA), } \\
\text { P. Pantaino (Italy) }\end{array}$ \\
\hline \multirow[t]{10}{*}{ 11:00-12:30 } & Platform Session VI (Large Trials - Acute Stroke) & $\begin{array}{l}\text { Chairpersons: H.P. Adams (USA), } \\
\text { Ch. Warlow (UK) }\end{array}$ \\
\hline & $\begin{array}{l}\text { Cytoprotective Effects of Neuropeptide Semax } \\
\text { (ACTH 4-10) in Acute Ischemic Stroke }\end{array}$ & V. Skvortsova, et al. (Russia) \\
\hline & $\begin{array}{l}\text { BAYx3702 in Acute Ischemic Stroke: Results from a } \\
\text { Phase II Dose Finding Study }\end{array}$ & P. Teal, et al. (Canada) \\
\hline & $\begin{array}{l}\text { The ATLANTIS rt-PA (Alteplase) Acute Stroke Trial: } \\
\text { Final Results }\end{array}$ & G.W. Albers, et al. (USA) \\
\hline & Heparin in Acute Embolic Stroke Trial (HAEST) & E. Berge, et al. (Norway) \\
\hline & $\begin{array}{l}\text { Does Breathing Supplemental } \mathrm{O}_{2} \text { Affect Outcome of } \\
\text { Acute Stroke? A Randomised Trial }\end{array}$ & O.M. Rønning, et al. (Norway) \\
\hline & $\begin{array}{l}\text { VENUS - Very Early Nimodipine Use in Stroke: } \\
\text { Final Results from a Randomised Placebo-Controlled Trial }\end{array}$ & J. Horn, et al. (The Netherlands) \\
\hline & $\begin{array}{l}\text { Preliminary Safety Report of a Randomized, Double-Blind, } \\
\text { Dose-Escalation Trial of Abciximab (ReoPro®) in Acute } \\
\text { Ischemic Stroke }\end{array}$ & H.P. Adams, et al. (USA) \\
\hline & $\begin{array}{l}\text { Final Results of the Second European-Australasian Acute } \\
\text { Stroke Study of Intravenous Alteplase in Acute Ischemic } \\
\text { Stroke (ECASS II) }\end{array}$ & C. Fieschi, et al. (Italy) \\
\hline & $\begin{array}{l}\text { Results of the GAIN } 2 \text { MRI Feasibility Study - A Substudy } \\
\text { of the Phase II } 2005 \text { Safety Trial of GV } 150526\end{array}$ & S. Warach, et al. (USA) \\
\hline 12:30-13:00 & Announcement of the 9th European Stroke Conference & $\begin{array}{l}\text { G.L. Lenzi (Italy), } \\
\text { F. Aichner (Austria) }\end{array}$ \\
\hline 13:00 & $\begin{array}{l}\text { Conference Close } \\
\text { Farewell Lunch }\end{array}$ & \\
\hline
\end{tabular}

